\title{
Barrierefreie Kommunikation als Voraussetzung und Mittel für die Partizipation benachteiligter Gruppen - Ein (polito-)linguistischer Blick auf Probleme und Potenziale von "Leichter" und "einfacher Sprache"
}

\author{
Bettina Bock (Leipzig)
}

\begin{abstract}
Leichte Sprache as the equivalent concept to Easy-to-read and a form of accessible communication has been established in the German-speaking countries since the early 2000s. It is addressed to people with learning disabilities and other groups with difficulties in reading and understanding (simple and complex) written text. In 2009 the German government ratified the Convention on the Rights of Persons with Disabilities and thereby committed itself to providing accessible information and communication. However, the concept's scientific exploration is still in its early stages. The existing lists of linguistic features and design rules were developed mainly intuitively. A systematic empirical investigation of Leichte Sprache is still to be done. The current practice is often slanted towards (over-)simplification, reduction of information, of lexical and grammatical variety and of stylistic differentiation.

Leichte Sprache is intended as a means of supporting and enabling participation in different areas of life for the target group. The article wants to investigate the chances and problems of this accessible form of communication as a prerequisite for and a means of political participation from a linguistic point of view. By way of example three texts are linguistically analysed and compared: For the elections to the Bundestag in 2013 the Social Democratic Party of Germany (SPD) released three manifestos, one regular version, one version modified into Leichte Sprache and one version modified into einfache Sprache, which is a similar concept allowing more complexity than Leichte Sprache. The linguistic analysis mainly focuses on the different strategies regarding the presentation of content, regarding lexis, adequate realisation of text type and function as well as on semantic and pragmatic aspects (frames, speech acts). Subsequently, the article discusses which consequences result from the different linguistic presentations with a view to enabling democratic participation.
\end{abstract}

\section{$1 \quad$ Einleitung}

"Leichte Sprache" ist eine Form barrierefreier Kommunikation ${ }^{1}$, die sich primär an Menschen mit Lernschwierigkeiten ${ }^{2}$ richtet. Im Bereich Politik ist sie nicht zuletzt deshalb mittlerweile

\footnotetext{
${ }^{1}$ Vgl. für einen linguistischen Überblick über barrierefreie Kommunikationsformen: Jekat et al. (2014). Linguistik Online 73, 4/15 - http://dx.doi.org/10.13092/lo.73.2196 
recht etabliert, weil sich die Bundesregierung mit der Ratifizierung der UNBehindertenrechtskonvention im Jahr 2009 zur Bereitstellung von Kommunikationsangeboten für benachteiligte Zielgruppen verpflichtet hat. In der Öffentlichkeit erfahren einzelne Texte in Leichter Sprache nicht selten Kritik, und zwar durchaus auch berechtigte, wie z. B. kürzlich im Fall der Leichte Sprache-Beilage zur Wochenzeitung Das Parlament, die sich - sprachlich ungewandt und verzerrend - mit den Anschlägen auf das französische Satiremagazin Charlie Hebdo beschäftigte (vgl. Stengel 2015).

Im Artikel soll aus politolinguistischer Perspektive erörtert werden, welche Potenziale der Ansatz Leichte Sprache für die Ermöglichung politischer Partizipation bei den entsprechenden Zielgruppen hat. Die derzeitige Praxis soll kritisch hinterfragt werden: Wird das partizipative Potenzial in leichten politischen Texten derzeit ausgeschöpft bzw. wird ihm überhaupt Bedeutung beigemessen? Wie müssen Texte in leichter Sprache gestaltet sein, damit die Adressaten angemessen informiert werden und selbstbestimmte Anschlusshandlungen möglich sind? Exemplarisch untersucht werden soll dies an drei Fassungen des SPD-Wahlprogramms aus der letzten Bundestagswahl (leicht, einfach und Vollversion): Welche Inhalte werden jeweils wie präsentiert? Welcher Wortschatz wird genutzt oder gemieden? Fragen der Textsortenspezifik werden ebenso berücksichtigt wie semantische und pragmatische Aspekte (Frames, Sprachhandlungen) sowie syntaktische Merkmle. ${ }^{3}$ An die Analysen schließt sich die Frage an, welche Folgen sich aus der jeweiligen sprachlichen Gestaltung für die Ermöglichung demokratischer Teilhabe ableiten lassen.

\section{Partizipation und Sprache}

\subsection{Sprache als Voraussetzung und Mittel für die Einforderung von Partizipation}

Die Frage nach dem Zusammenhang von Sprache und Demokratie betrifft zu einem großen Teil die Frage nach der Funktion von Kommunikation und Information für demokratische Partizipation und Entscheidungsprozesse:

Kommunikation und Information sind also die Grundkategorien der westlichen DemokratieAuffassung. Sie ermöglichen jene diskursive Meinungsbildung, auf der demokratische Entscheidungs- und Handlungsvollzüge aufruhen [sic.].

(Jäger 1996: 46)

In diesem Artikel soll es vorrangig um die Frage gehen, welche Rolle Sprache und Kommunikation für die Ermöglichung und Realisierung von Partizipation spielen: Zunächst einmal ist intuitiv einsichtig, dass die Teilhabe an demokratischen Prozessen in unterschiedlichen Gesellschaftsbereichen zu einem Großteil über (sprachliche) Kommunikation läuft. Als Beispiele für die öffentliche Thematisierung dieses Zusammenhangs könnte man die immer wiederkehrende Kritik an schwer verständlicher Sprache in einzelnen Lebens- und Kommunikationsbereichen anführen (Rechtssprache, Verwaltungssprache, Politikersprache) sowie die damit verbundene Forderung nach mehr Verständlichkeit und Transparenz. Auch bei politischen Entscheidungen werden zu einzelnen Anlässen Transparenz- und Mitspracheforderungen formu-

\footnotetext{
${ }^{2}$ Selbstgewählte Bezeichnung als Alternative zu "Menschen mit geistiger Behinderung", der aufgrund semantischer Unschärfe in der förderpädagogischen Forschung aber tlw. mit Skepsis begegnet wird.

${ }^{3}$ Aspekte der Typografie sowie Bilder wurden in die Analyse nicht einbezogen.
} 
liert. Über Kommunikation wird also Partizipation (zu einem großen Teil) realisiert, und gleichzeitig ist Kommunikation der Weg, diese einzufordern. Partizipationsmöglichkeiten und -formen werden sprachlich verhandelt. Zu einer Hürde kann dies für Gruppen werden, die von vornherein von (insbesondere schrift- und bildungs-)sprachlicher ${ }^{4}$ Kommunikation ausgeschlossen sind oder nur begrenzten Zugang dazu haben. Wer sich in einer Demokratie nicht oder kaum Zugang zu Information und Kommunikation verschaffen kann und sich außerdem (als Folge) nur eingeschränkt an gesellschaftlichen Debatten beteiligen kann, dessen aktive und passive Partizipationsmöglichkeiten sind beschnitten.

Eine wichtige Voraussetzung für die passive politische und rechtliche Inklusion von Menschen mit Behinderung sind zunächst einmal die formalen Rechte und Chancen (u. a. geregelt durch das Behindertengleichstellungsgesetz, UN-Behindertenrechtskonvention, SGB IX) (vgl. Wansing 2005: 170). Die Realisierung von Partizipation hängt jedoch wesentlich von der tatsächlichen Aktivierung von Interessensartikulationen und -durchsetzungen ab (aktive politische Inklusion) (vgl. ebd.). Sprache und Kommunikation kommt hier eine Schlüsselfunktion zu: Einerseits wird das Wissen um Rechte und Partizipationsmöglichkeiten sprachlich vermittelt. Der Zugang zu schriftlichen Texten ermöglicht ein selbständiges Aufsuchen von Informationen. Insofern kann der Zugang zu (Schrift-)Sprache als ein wichtiges Element für die Erlangung von Selbstbestimmung und Teilhabe gelten. 5 Andererseits muss dieser Zugang, d. h. Barrierefreiheit, an den Stellen eingefordert werden, wo eine Realisierung entsprechender Angebote noch aussteht - ebenfalls mit den Mitteln von Sprache und Kommunikation. Das heißt, dass ein gewisses Maß an sprachlichen, kommunikativen und literalen Kompetenzen sowohl Voraussetzung für Selbstbestimmung und Partizipation ist, als auch ein Mittel, um diese einzufordern, durchzusetzen, Protest zu bekunden usw. Wer von dem Zugang zu Information und Kommunikation völlig ausgeschlossen ist, hat demnach auch nur geringe Möglichkeiten, ihn einzufordern. Behinderung, und insbesondere geistige Behinderung, stellt einen der Hauptrisikofaktoren für Exklusion und kumulierte Ausgrenzung dar (vgl. Wansing 2005: 78).

\subsection{Leichte Sprache: Eine Form barrierefreier Kommunikation}

Die Ursprünge der deutschsprachigen Ansätze (darunter sollen hier neben der Leichten Sprache auch ähnliche Konzepte wie "Leicht Lesen" oder "einfache Sprache" zählen) liegen in der US-amerikanischen Empowerment-Bewegung People First, die sich als Selbstvertretungsorganisation von Menschen mit Lernschwierigkeiten in den 70er Jahren organisierte. Das Phänomen Leichte Sprache hat also selbst eine "partizipative Entstehungsgeschichte" und ist intendiert als ein Mittel der Zugänglichmachung von Kommunikation und Information für Menschen, die schon mit dem Lesen und Verstehen alltäglicher Texte Schwierigkeiten haben. Die Adressaten Leichter Sprache sind wie bei anderen Formen barrierefreier Kommunikation (bspw. Gebärdensprache, Braille-Schrift, Audiodeskription) auf diese Form der Informations-

\footnotetext{
${ }^{4}$ Bildungssprache ist hier nicht normativ zu verstehen, sondern soll mit Feilke (2012: 5) gefasst werden als "die besonderen sprachlichen Formate und Prozeduren einer auf Texthandlungen wie Beschreiben, Vergleichen, Erklären, Analysieren, Erörtern usw. bezogenen Sprachkompetenz, wie man sie im schulischen und akademischen Bereich findet" sowie die entsprechenden grammatischen Mittel.

${ }^{5}$ Selbstverständlich können Informationen auch mündlich vermittelt werden, das wäre eine Aufgabe bspw. der persönlichen Assistenzen. Literale Kompetenzen bringen allerdings eine größere Unabhängigkeit mit sich.
} 
aufbereitung angewiesen. 2001 gründete sich eine entsprechende Organisation "Mensch zuerst" in Deutschland. Bereits in dieser Zeit entstand das erste Wörterbuch in Leichter Sprache, 2006 wurde das Netzwerk Leichte Sprache gegründet, das sich seitdem verstärkt für entsprechende barrierefreie Kommunikationsangebote einsetzt. Mittlerweile kann diese Form barrierefreier Kommunikation als relativ etabliert gelten. Es existiert eine große Anzahl von (sprachlich teilweise sehr verschieden gestalteten) Texten und Informationsangeboten. Die Bundesregierung hat sich mit der Ratifizierung der UN-Behindertenrechtskonvention 2009 dazu verpflichtet, flächendeckend barrierefreie Informationen zur Verfügung zu stellen, was sich beispielsweise in den durchgängig vorhandenen leichten Fassungen der Webseiten aller Bundesinstitutionen zeigt. Auch alle großen Parteien stellen mittlerweile Unterlagen und Webseiten in Leichter Sprache bereit, insbesondere Wahlkampf-Texte.

In der Praxis der Leichten Sprache gibt es verschiedene Akteure und Arbeitsprinzipien. Die Qualität der Texte ist teilweise recht unterschiedlich. Am häufigsten wird (explizit oder implizit) auf den frei zugänglichen Regelkatalog des Netzwerks Leichte Sprache Bezug genommen (Netzwerk Leichte Sprache 2013). ${ }^{6}$ Die dort formulierten Empfehlungen lassen eine relativ eindimensionale Tendenz zur Vereinfachung, Reduzierung und Selektion (von Information, von Wortschatz, von syntaktischer Variation, von Formulierungsvielfalt usw.) erkennen. Dies wird sich auch in der genaueren Betrachtung der Wahlprogrammfassungen (Kapitel 4) zeigen. Bestimmte sprachliche Merkmale (repetitive Syntax, bestimmte sprachliche Formeln, Satzlänge, Anlehnung an umgangssprachliche Register und tlw. mündlichen Sprachgebrauch usw.) und typografische Eigenschaften (Schriftart Arial in Großdruck, farbige Illustration, seltener Icons, optische Worttrennung) dürften mittlerweile als Signal- und Erkennungszeichen für Leichte Sprache fungieren. Die Diskrepanz besteht darin, dass mit der Reduktion der sprachlichen Mittel keine Reduktion der Funktionalität einhergeht (wie es beispielsweise bei Subsprachen/Fachsprachen der Fall ist): Die kontextunabhängig formulierten Regeln Leichter Sprache sollen - ihrer Intention und der Ansicht vieler Akteure in der Praxis nach - in allen Kommunikationsbereichen und Textsorten verbesserte Verständlichkeit ermöglichen (zu dieser Problematik vgl. Bock 2014: 20-25). Ein weiteres Problemfeld ist das zugeschriebene geringe Ansehen einer solchen reduzierten Sprachform (vgl. ebd.: 24-26), das sich seit der zunehmenden Präsenz von leichten Texten auch in der öffentlichen Diskussion niederschlägt (vgl. NZZ 2014; Liessmann 2014). Darauf wurde in der stetigen Weiterentwicklung des Konzepts bisher nicht substanziell reagiert. Diese Problematik ist m. E. von zentraler Bedeutung, bildet aber ein eigenes Themenfeld, das im Folgenden nur am Rande Erwähnung finden wird. Die wissenschaftliche Auseinandersetzung mit dem Praxisphänomen Leichte Sprache steht derzeit noch relativ am Anfang: Bisher sind es die Erziehungswissenschaften, Soziologie, Übersetzungswissenschaft und zunehmend auch Linguistik und Sprachdidaktik, die sich mit dieser Form barrierefreier Kommunikation beschäftigen. ${ }^{7}$

\footnotetext{
${ }^{6}$ Manche Texte wählen auch ohne erkennbaren Bezug die Bezeichnung Leichte Sprache als Label.

${ }^{7}$ Vgl. beispielhaft: Aus Politik und Zeitgeschichte (2014); Jekat et al. (2014); BMAS-Projekt "Leichte Sprache im Arbeitsleben" an der Universität Leipzig (http://research.uni-leipzig.de/leisa/).
} 


\section{3 (Politische) Partizipation und Inklusion: Begriffsklärungen}

Partizipation kann als zentrales Merkmal von Demokratie gelten. Gemeint ist hier: politische Partizipation bzw. die Teilhabe an (verwaltungs-)politischen (Entscheidungs-)Prozessen. Die Begriffe "Partizipation" und "Teilhabe" werden in Definitionen häufig synonym gebraucht (so bspw. Schubert/Klein 2011: 223). Teilweise führt die Vagheit der Definitionen aber auch dazu, dass Wörterbücher nahelegen, es handle sich um zwei verschiedene Phänomene - so bspw. in Theunissen, Kulig und Schirbort (2013), die die Begriffe in je einem eigenen Wörterbucheintrag erklären, ohne auch nur ansatzweise eine Abgrenzung vorzunehmen. Es scheint so zu sein, dass im politikwissenschaftlichen Kontext der Begriff Partizipation verbreiteter ist, ${ }^{8}$ während in der Inklusions- und Teilhabeforschung und in angrenzenden Disziplinen der Teilhabebegriff prominenter ist und dort auch nicht nur bzw. vorrangig auf politische Partizipation/Teilhabe eingegrenzt wird. Die WHO definiert Teilhabe sehr allgemein als Einbezogensein in eine Lebenssituation (vgl. WHO-ICF 2005: 19). In einem Diskussionspapier der Deutschen Vereinigung für Rehabilitation und der Deutschen Gesellschaft für Rehabilitationswissenschaften wird dies spezifiziert:

Teilhabe ist dann gegeben, wenn eine Person sozial eingebunden ist, d. h. wenn individuelle und umweltbezogene Faktoren es ermöglichen, dass die Person die sozialen Rollen, die ihr wichtig und ihrer Lebenssituation angemessen sind (z. B. in der Familie, im Beruf, in der sozialen, religiösen und politischen Gemeinschaft), auch einnehmen und zu ihrer Zufriedenheit ausfüllen kann.

(DVfR/DGRW 2012: 2)

Verbreitet ist außerdem der überdachende Begriff der gesellschaftlichen Teilhabe (vgl. Wansing 2005). Politische Partizipation spielt in der Teilhabeforschung eine untergeordnete Rolle, wie Wansing (2005: 82) kritisiert: In soziologischen Analysen stehe "nach wie vor das Thema Arbeit im Vordergrund, während die soziale, kulturelle und politische Partizipation immer noch wenig Berücksichtigung findet". Durch die fehlende konzeptionelle Grundlegung des Teilhabebegriffs sieht Wansing (2005: 17) außerdem die Gefahr, dass er vage und unspezifisch interpretiert wird und damit an Wirkkraft verlieren kann. ${ }^{9}$

In der Öffentlichkeit thematisiert wird politische Partizipation in aller Regel dann, wenn die Teilhabe der Bevölkerung an politischen Willensbildungsprozessen oder Verwaltungsentscheidungen umstritten ist oder eingefordert wird. Bora (2005: 20) kennzeichnet Partizipation als "semantische Formel, mit der Inklusionsverhältnisse thematisiert werden" und damit die Frage, inwiefern Menschen an Organisationen und Verfahren im (weitgefassten) Bereich Politik (durch Mitgliedschaft, Mitwirkung, Beratung usw.) teilhaben können oder davon ausgeschlossen sind (vgl. ebd.: 22). "Wo Partizipation thematisch wird, weist dies darauf hin, dass Inklusion [...] umstritten ist und eingefordert wird", so Bora (2005: 23). Inklusion bezieht sich im Gegensatz zur häufig verkürzend assoziierten Bedeutung 'Inklusion von Menschen mit Behinderung' eben nicht nur auf diese eine Gruppe, "sondern sie hat ebenso andere Grup-

\footnotetext{
${ }^{8}$ Bora (2005: 15) vertritt sogar aus einem spezifischen Blickwinkel die Ansicht, dass Partizipation immer als politische Partizipation zu verstehen sei, da "der Begriff der Partizipation selbst bereits als politische Inklusionsformel interpretiert wird".

${ }^{9}$ In Forschung und Politik wird eine Konkretisierung und Operationalisierung von Teilhabe gefordert und angestrebt (vgl. Diskussionspapier Teilhabeforschung 2012). Im Sinne partizipativer Forschung wird dabei auch die Beteiligung der Betroffenen an der Erforschung und Theoriebildung in diesem Gebiet gefordert (ebd.: 6).
} 
pen im Blick, die allzu leicht marginalisiert, ausgegrenzt und benachteiligt werden" (Theunissen/Schwalb 2012: 17-18).

Die gesamte wissenschaftliche Diskussion von Teilhabechancen für Menschen mit Behinderung steht derzeit unter der Leitidee von Inklusion und der Überwindung der sogenannten Zwei-Welten-Theorie. Die Trennung in eine Welt der Nicht-Behinderten und eine Welt der Behinderten soll überwunden werden durch die Sichtweise, dass eine Vielzahl von heterogenen Lebenswelten (mit je individuellen Inklusionsprofilen und Exklusionsrisiken) existiert (vgl. Theunissen/Schwalb 2012: 14, 20-21). Inklusion als Leitbegriff beschreibt das Recht auf Zugehörigkeit, Selbstbestimmung und Partizipation (vgl. ebd.: 17) und grenzt sich von dem Begriff der Integration ab. Dieser wurde reduziert auf strukturelle Eingliederung (z. B. räumliche Integration) und erhebt Partizipation am gesellschaftlichen Leben noch nicht zum zentralen Ziel (vgl. ebd.: 14-15). Angestrebt wird nun stattdessen ein "größtmögliches Maß an Inklusion und Partizipation" (ebd.: 23). Die Zwei-Welten-Situation soll überwunden werden, d. h. allen soll der weitgehende Zugang zu ein und derselben Welt ermöglicht werden. Seinen Niederschlag findet das u. a. im Konzept Barrierefreiheit. In der inklusiven Perspektive ist eine Gesellschaft dafür verantwortlich, die nötigen Voraussetzungen für Teilhabe und Inklusion zu schaffen: Strukturen und Institutionen müssen so verändert werden, dass sie den Rechten, Interessen und Bedürfnissen aller Mitglieder einer Gesellschaft entsprechen können:

[A]llen Mitgliedern einer Gesellschaft [müssen] wichtige soziale und kulturelle Systeme (z. B. allgemeiner Bildungseinrichtungen und Dienstleistungen, Arbeitsplätze in regulären Betrieben) verfügbar und zugänglich sein.

(Theunissen/Schwalb 2012: 18)

Den rechtlichen Rahmen dafür bilden z. B. die Antidiskriminierungsgesetze. In der UNBehindertenrechtskonvention wird der Zugang zu Information und Kommunikation explizit als Voraussetzung für "eine unabhängige Lebensführung und die volle Teilhabe in allen Lebensbereichen" (Art. 9, Abs. 1) angeführt. Artikel 29 regelt die Ermöglichung politischer Partizipation, insbesondere Wahlen und Mitwirkungsmöglichkeiten:

Die Vertragsstaaten garantieren Menschen mit Behinderungen die politischen Rechte sowie die Möglichkeit, diese gleichberechtigt mit anderen zu genießen, und verpflichten sich,

a) sicherzustellen, dass Menschen mit Behinderungen gleichberechtigt mit anderen wirksam und umfassend am politischen und öffentlichen Leben teilhaben können, [...]

b) stellen sie sicher, dass die Wahlverfahren, -einrichtungen und -materialien geeignet, zugänglich und leicht zu verstehen und zu handhaben sind; [...]

c) aktiv ein Umfeld zu fördern, in dem Menschen mit Behinderungen ohne Diskriminierung und gleichberechtigt mit anderen wirksam und umfassend an der Gestaltung der öffentlichen Angelegenheiten mitwirken können

(UN-Behindertenrechtskonvention, Art. 29)

Eine inklusive Gesellschaft, gedacht als vollständiger Einbezug von allen Menschen mit Lernschwierigkeiten, ordnen Theunissen und Schwalb (2012: 22) allerdings als visionäre Vorstellung ein. Sie stellen in Frage, dass solch eine Gesellschaft erreicht werden kann. Inklusion sei in jedem Fall immer nur auf der Ebene von Teilsystemen möglich, auf dieser müsste auch das Problem der Exklusion verhandelt werden (ebd.: 23). Systemtheoretisch gesprochen sind in der funktional differenzierten modernen Gesellschaft Individuen ohnehin nur teil- und zeitweise in Funktionswelten integriert (vgl. Wansing 2005: 39; zur Kritik an der rein deskrip- 
tiven systemtheoretischen Perspektive auf Exklusion vgl. ebd.: 48, 51). In diesem Sinne ist jeder Einzelne immer auch ausgeschlossen und vollständige Inklusion unmöglich. Dennoch gibt es natürlich problematische Exklusionsprozesse und Exklusionsrisiken, die maßgeblich negativen Einfluss auf die Lebensgestaltung und den Lebenslauf des Einzelnen haben können. Inklusion zielt auf die Angleichung von Inklusions- und Partizipationschancen.

\section{Eine kritische linguistische Sicht auf Leichte Sprache}

In der öffentlichen Diskussion werden dem Konzept Leichte Sprache sehr hohe Erwartungen (und auf der anderen Seite ebenso viel Kritik) entgegengebracht: Vom Anspruch her wird Leichte Sprache - wie bereits eingangs erwähnt - häufig als ein Ansatz verstanden, mit dem sämtliche Texte in allen Lebens- und Kommunikationsbereichen in eine verständliche Sprache "übersetzt" werden können, ${ }^{10}$ die - so eine weitere verbreitete Annahme - letztendlich für alle den Zugang zu auch komplexen Inhalten erleichtert. ${ }^{11}$ Leichte Sprache erscheint in dieser Sicht als Universallösung für alle Verständlichkeitsprobleme. Aber die Umsetzung und die Auswahl sprachlicher und gestalterischer Prinzipien ist zweifellos komplexer als derzeitige Regelkataloge suggerieren.

Versteht man Leichte Sprache als Instrument zur Ermöglichung von Partizipation und Selbstbestimmung, fehlt der eben beschriebenen, derzeit dominanten Sichtweise ein ganz entscheidendes Element: die Funktion, (sprachliche) "Brücken" zu Kommunikations- und Lebensbereichen zu "bauen". Betrachtet man die derzeitige Leichte Sprache aus varietätenlinguistischer Perspektive, erscheint sie als eine "Parallelvarietät", die vom Anspruch her alle anderen Varietäten überdacht und meint, alles leisten zu können, was diese auch leisten. ${ }^{12} \mathrm{Ob}$ fachsprachliche oder alltagssprachliche, ob Texte mit konkreten oder abstrakten Inhalten, ob Texte, die spezifische oder allgemeine Adressatengruppen ansprechen, ob Gesetzestext, Arbeitsvertrag, Psalm oder Bedienungsanleitung - alle könnten (völlig ohne Bedeutungs- oder inhaltliche Verluste, so eine verbreitete Vorstellung,) in die derzeitige Leichte Sprache übertragen werden, und zwar mit dem Ergebnis größerer Verständlichkeit und Zugänglichkeit. Leichte Sprache erscheint in dieser Sichtweise als Varietät, die parallel zu allen anderen existiert, die alle Funktionen der vielfältigen Textsorten potenziell zu realisieren imstande ist (und damit universellen Zugang verschafft) und die dennoch nur auf eine Auswahl an ganz bestimmten sprachlichen Mitteln zurückgreift, die nicht kontextspezifisch differenziert werden: Multifunktionalität und universelle Anwendbarkeit stehen auf der einen und Restringiertheit in den sprachlichen Formen auf der anderen Seite (vgl. ausführlicher dazu Bock 2014: 20-21). Nicht erst in der Praxis wird hier m. E. ein großer Widerspruch sichtbar.

Wie die Analyse noch genauer zeigen wird, ist die Umsetzung der Idee Leichter Sprache derzeit tatsächlich häufig mangelhaft, was seine Ursache auch in der fehlenden theoretischen Fundierung des Ansatzes hat sowie in der noch ausstehenden empirischen Überprüfung, auch durch die Linguistik. Die Vermittlungs- und Brückenaufgabe, die leichten Texten eigentlich

\footnotetext{
${ }^{10}$ Sprachliche Regeln und Empfehlungen werden nicht kontextspezifisch differenziert.

${ }^{11}$ In den Regeln des Netzwerks Leichte Sprache (2013: 2) heißt es: "Leichte Sprache verstehen alle besser".

${ }^{12} \mathrm{Zu}$ einer detaillierteren Einordnung in das Varietätenspektrum vgl. Bock 2014: 28-39. Leichte Sprache wird teilweise fälschlicherweise als Gruppenvarietät (also Sprachgebrauch und Erkennungszeichen einer sozialen Gruppe) missverstanden, zu verstehen ist sie vielmehr als Vermittlungsvarietät.
} 
zukommt, spielt sehr häufig kaum eine Rolle. Immer wieder gibt es Texte, deren Funktion und Ziel völlig unklar bleibt (für eine Beispielanalyse vgl. Bock 2014: 41-43). Typischerweise werden dann allgemeine Informationen zu einem bestimmten Thema zusammengetragen, ohne dass klar wird, was der Leser mit dem Wissen eigentlich anfangen soll (Textfunktion, Anschlusshandlungen), wozu es gut ist oder woran anknüpfbar (Kontext). Gerade im Kommunikationsbereich Politik haben etliche Texte in Leichter Sprache eher eine "Aushängeschildfunktion", als dass sie das Ziel erkennen lassen, den Lesern komplexe Inhalte so zu vermitteln, dass sie selbstbestimmt handeln können. Mit "Aushängeschildfunktion" meine ich, dass die Bereitstellung leichter Texte als Signal für bestimmte politische Überzeugungen dient; sie soll sagen: "wer solch ein Kommunikationsangebot macht, setzt sich für Inklusion/Barrierefreiheit/Partizipation/... ein". Problematisch wird dies, wenn die Funktion als Aushängeschild die primären Textfunktionen dominiert und die Vermittlungsaufgabe vernachlässigt wird (vgl. Analyse in Kapitel 4; für weitere Beispiele vgl. Bock in Vorbereitung).

In der oben gewählten Bezeichnung "Parallelvarietät" drückt sich auch bereits die potenzielle Gefahr aus: Viele leichte Texte verschaffen tatsächlich nur Zugang zu einer leichten Parallelwelt, nicht aber zur "allgemeinen" (Text-)Welt. Zugespitzt formuliert läuft die Idee damit Gefahr, die Zwei-Welten-Situation eher zu manifestieren als zu überwinden: Leichte Texte schaffen es häufig zu wenig, ihren Lesern einen Anschluss an die Inhalte der schweren Ausgangstexte, an allgemeine öffentliche Debatten usw. zu eröffnen und ihnen so auch den Weg zu mehr Teilhabe zu ebnen. Wer beispielsweise das in Kapitel 4 analysierte SPDWahlprogramm ausschließlich in der Leichte Sprache-Fassung liest, bekommt zwar Informationen zu den politischen Zielen der SPD, es bleibt allerdings mehr als fraglich, dass er mit diesem Wissen einen auch nur rudimentären Zugang zu aktuellen politischen Debatten und Fragen hat. Meines Erachtens läuft Leichte Sprache hier Gefahr, selbst eine Sprachbarriere zu werden und die Abkopplung von der in schwerer Sprache verfassten Welt nur zu verschleiern (vgl. Bock i. E.). Partizipation kann so eventuell sogar erschwert werden. Die Intention von "Mensch zuerst", von Netzwerk Leichte Sprache und anderen Akteuren ist zweifelsfrei eine andere. Es bedarf daher einer kritischen Überprüfung des Konzepts Leichte Sprache und einer Weiterentwicklung. Diese zunächst sehr kritische Einschätzung soll in den folgenden Textanalysen zunächst überprüft und differenziert werden: In welchem Maße schöpfen Leichte Sprache und verwandte Ansätze die Potenziale, die sie zweifellos haben, aus, um Barrierefreiheit für Menschen mit Lernschwierigkeiten herzustellen und den Weg zu Partizipation zu ebnen? Diese Frage soll im Folgenden aus sprachwissenschaftlicher Perspektive an der Analyse der drei Wahlprogramm-Fassungen erörtert werden.

\section{Analyse der Wahlprogrammfassungen}

Die SPD hat ihr Bundestagswahlprogramm 2013 in drei Fassungen veröffentlicht: In einer regulären Version, einer Version in Leichter Sprache und einer in einfacher Sprache. Die leichte Fassung wurde vom AWO Büro für Leichte Sprache erstellt, die einfache Fassung stammt von der Agentur Klar \& Deutlich. Anders als Leichte Sprache existiert für einfache Sprache kein gleichermaßen kodifizierter Regelkatalog. Sie richtet sich aber an ähnliche Zielgruppen. ${ }^{13}$ Meist wird dieses Label benutzt - und so ist es auch im Fall des Wahlprogramms -

\footnotetext{
${ }^{13}$ Eine Gegenüberstellung und Charakterisierung der beiden Ansätze findet sich in Bock 2014: $20-26$.
} 
, um zu signalisieren, dass der Text sprachlich komplexer gestaltet ist als Texte in Leichter Sprache. In programmatischer Weise stellt das SPD-Wahlprogramm (VV) die Bedeutung des Zugangs zu "qualitativ hochwertiger Information" für die demokratische Gesellschaft und Partizipation heraus:

Voraussetzung für eine demokratische und offene Gesellschaft sind demokratische Kultur und Öffentlichkeit genauso wie Transparenz und Partizipation. Dazu gehört auch der Zugang für alle zu qualitativ hochwertiger Information. Zugang zu allen Informationsmöglichkeiten ist ein demokratisches Bürgerrecht.

(Wahlprogramm-VV: 96)

Auch vor diesem Hintergrund sollen die beiden vereinfachten Wahlprogramm-Fassungen im Folgenden verglichen werden. Die Analyse beschränkt sich weitgehend auf die Kapitel zur Arbeitspolitik und zeichnet Modifizierungsprinzipien von leichter und einfacher Fassung exemplarisch nach.

\subsection{Wortwahl und Schlagwörter}

Einen ersten, wenngleich rein strukturellen, vergleichenden Einblick in den Wortschatz der drei Wahlprogramm-Fassungen können quantitative Parameter geben: Token-Anzahl und Type-Token-Ratio (TTR), mit der die Wortvariation berechnet wird, gelten traditionell als Indizien für die Schwierigkeit von Texten. Interessant ist hier zunächst, dass die einfache Fassung des Wahlprogramms, die sich an Leser mit höheren Lesekompetenzen richtet als die leichte Fassung, weniger Token umfasst als der Leichte Sprache-Text (2503 Token gegenüber 3362 Token). Auch bei der TTR schneidet die einfache Fassung mit einem deutlich geringeren Wert ab (2,87\%) als die leichte $(4,65 \%)$. Der Wortvariationswert der WahlprogrammVollfassung liegt nicht sehr viel höher (5,12\%). Geringe Wortvariation im Text wird interpretiert als Anzeichen für Verständlichkeit/Zugänglichkeit (auf Wortschatzebene), was hier zu dem überraschenden Ergebnis führt, dass die leichte Fassung unwesentlich weniger zugänglich ist in Bezug auf den Wortschatz als die Vollfassung, während die einfache Fassung die größte Verständlichkeit verzeichnen kann. Dazu muss aber angemerkt werden, dass die Vollfassung schon aufgrund ihrer Länge (41.930 Token) und auch aufgrund ihres häufig fachsprachlichen Wortschatzes hinsichtlich Verständlichkeit und Rezipierbarkeit kaum mit dem Wahlprogramm in Leichter Sprache gleichgesetzt werden kann. Die Parameter geben also höchstens einen ersten Eindruck von den Texten wieder.

Welchen Wortschatz verwenden die drei Fassungen? Ein interessanter Aspekt ist die Verwendung von Schlagworten. ${ }^{14}$ Insbesondere in der leichten Wahlprogrammfassung werden zentrale Schlagworte konsequent gemieden. Nun kann man das Fehlen von (Schlag-)Phrasen und Floskeln durchaus als positiv einstufen. Formulierungen wie "Anstrengung und Fleiß müssen sich für die Menschen wieder lohnen; verantwortliche Politik im Interesse der Menschen" (SPD-Wahlprogramm VV, 17); "dass sich Leistung lohnen muss; Angleichung der Lebens- und Arbeitsbedingungen [und Renten, B. B.] in Ost- und Westdeutschland" (ebd.: 19); "gleicher Lohn für gleiche Arbeit" (ebd.: 20) fehlen in einfachem und leichtem Wahlpro-

14 Erfasst werden Wörter mit programmatischem sowie Meinungsgehalt, die sich gleichzeitig durch einen gewissen "semantischen Spielraum" sowie Umstrittenheit und Gruppengebundenheit auszeichnen (vgl. Schröter/Carius 2009: 20). 
gramm. Jedoch ist die konsequente Nicht-Erwähnung politischer Schlagworte auch problematisch, da sie auf ganz bestimmte Inhalte, Themen und Debatten verweisen, die mit dem Fehlen der Schlagworte nicht mehr zur Sprache kommen. In den untersuchten Kapiteln zur Beschäftigungs- und Arbeitsmarktpolitik aus dem SPD-Wahlprogramm (VV) spielen Begriffe und Konzepte wie Mindestlohn, Niedriglohn(sektor), Leiharbeiter, Mini-Job, ScheinArbeitsverträge, Schein-Selbständigkeit, (Langzeit-)Arbeitslosigkeit, sozial abgesicherte Arbeit, (mehr) Demokratie im Betrieb eine tragende Rolle - allesamt Konzepte, die eine unmittelbare Alltagsrelevanz auch für Menschen mit Lernschwierigkeiten im Arbeitsleben haben (können). Mit weiteren Schlagwörtern werden abstraktere Konzepte bezeichnet, beispielsweise das Gesamtziel der SPD: "Vollbeschäftigung in guter Arbeit", außerdem "Stärkung des Tarifsystems" oder die Unterscheidung zwischen "prekären Arbeitsverhältnissen" und "Normalarbeitsverhältnissen". Alle diese Schlagwörter kommen in der leichten Wahlprogrammfassung nicht vor, obwohl sie für die Zielgruppe relevant sind und sich durch Bezug auf konkrete Arbeitssituationen und -verhältnisse veranschaulichen ließen. Ebenso verhält es sich mit den Schlagworten Inklusion/inklusiv, Mitbestimmung, Selbstbestimmung, Teilhabe oder Partizipation (auch Demokratie/demokratisch). Sie werden in der leichten Wahlprogrammfassung erstaunlicherweise nicht verwendet. In der Broschüre in einfacher Sprache werden einige der Schlagworte gebraucht (Mindestlohn, Leiharbeiter, Mini-Job) und ausdrücklich und sehr aussagekräftig erklärt. Gute Arbeit, Tariflöhne für alle und sichere Verträge lehnen sich an Schlagworte der Wahlprogramm-Vollfassung an; Inklusion und verwandte Themen werden im einfachen Sprache-Text nicht aufgegriffen, weshalb die entsprechenden Schlagwörter fehlen.

Wie werden die genannten Konzepte nun stattdessen thematisiert, oder kommen sie in den modifizierten Fassungen tatsächlich nicht zur Sprache? Das Kapitel, das im Wahlprogramm in Leichter Sprache die arbeitsmarkt- und beschäftigungspolitischen Inhalte zusammenfasst, trägt den Titel "Alle sollen einen guten Lohn bekommen" (SPD-Wahlprogramm LS: 8). Tatsächlich werden alle vorgestellten Ziele im Bereich Arbeitspolitik an den Frame 'Lohn' bzw. den übergeordneten Frame 'Beschäftigungsverhältnis' (im FrameNet15 'Being_employed') gebunden. Es findet hier also eine Konkretisierung durch Reduktion statt, indem die Vielzahl politischer Themen und Forderungen auf einen Themenbereich eingegrenzt wird (Lohn, Beschäftigungsverhältnis), der zudem eine unmittelbare Relevanz für potenzielle Leser aufweist. Das Schlagwort gute Arbeit wird semantisch in der leichten Fassung auf die Teilaspekte Bezahlung und "feste Arbeitsplätze" (gemeint sind sozialversicherungspflichtige Beschäftigungsverhältnisse) reduziert: "Die Arbeit soll gut bezahlt werden". (ebd.). Als zweites Ziel wird (ohne das Schlagwort Mindestlohn oder ähnliche Formulierungen zu gebrauchen) angeführt:

Jeder Mensch bekommt mindestens 8,50 Euro in der Stunde.

Am besten aber mehr.

Jeder Mensch, der arbeitet, soll genug Geld zum Leben haben.

(SPD-Wahlprogramm LS: 8)

15 Auf semantischen Frames basierendes Online-Wörterbuch des Englischen. 
In der einfachen Fassung findet man eine ähnliche Konkretisierungsstrategie: Die Fülle an arbeitsmarktpolitischen Zielen wird ebenfalls auf die Frames 'Lohn' und 'Beschäftigungsverhältnis' "eingedampft". Allerdings wird dies verbunden mit der expliziten Erklärung zentraler Schlagworte, was dem Leser ermöglicht, am allgemeinen öffentlichen Diskurs teilzunehmen und ihm die thematischen Schnittstellen offensichtlich macht. Leiharbeit wird beispielsweise in einer typografisch abgesetzten Spalte explizit erklärt: "Braucht ein Betrieb für kurze Zeit mehr Arbeitskräfte? Dann kann er sie sich bei einer Leiharbeitsfirma "ausleihen". Diese Firma vermittelt Arbeitskräfte" (SPD-Wahlprogramm ES: 7).

Mindestlohn wird im Fließtext und unter eigener Überschrift paraphrasiert und als Ziel begründet: "Alle sollen mindestens 8,50 Euro pro Stunde verdienen. Mit weniger kommen die Menschen nicht aus. Wer arbeitet, muss genug Geld für Essen, Miete und Strom haben" (ebd.).

Das Thema "gerechte Entlohnung", das im SPD-Wahlprogramm eine Rolle spielt (und das als Teil des Konzepts 'gute Arbeit' gelten kann), wird in den beiden vereinfachten Fassungen folgendermaßen formuliert:

(1) Gleichen Lohn für Frauen und Männer

Frauen verdienen im Durchschnitt 22 Prozent weniger als Männer. Wir wollen im Gesetz festlegen lassen: Frauen und Männer sollen für dieselbe Arbeit den gleichen Lohn bekommen.

(SPD-Wahlprogramm ES: 8)

(2) Für die gleiche Arbeit soll jede Frau und jeder Mann

den gleichen Lohn bekommen.

Egal, ob man in einer Firma einen festen Arbeitsplatz hat.

Oder ob man in einer Firma für eine kurze Zeit aushilft.

(SPD-Wahlprogramm LS: 8)

Im direkten Vergleich fällt auf, dass im einfachen Text (1) im Vergleich zu (2) mehr (Kontext-)Informationen gegeben werden (geringerer Durchschnittsverdienst, neues Gesetz), während in der leichten Fassung der Ausdruck "gleiche Arbeit" näher spezifiziert wird. Umschrieben werden in (2) (vermutlich) Konzepte wie "Leiharbeit" oder "Mini-Job" (in einer Firma für kurze Zeit aushelfen). Die Allgemeinheit der Umschreibungen und das Fehlen der Schlagwörter lässt aber kaum genauere Interpretationen oder Äquivalenzbestimmungen zu. In (1) wird außerdem ein Begründungszusammenhang hergestellt, der in der leichten Fassung fehlt.

Relativ ausführlich wird das Thema "gute/sozialversicherungspflichtige Arbeitsverhältnisse" in der einfachen Fassung erklärt, begründet und als sinnvolles Ziel dargestellt, und zwar unter dem Schlagwort sichere Verträge (ebd.: 7-8). Die leichte Fassung fasst das Thema unter dem Begriff feste Arbeitsplätze, wobei diese Formulierung offenkundig mit sozialversicherungspflichtigen Beschäftigungsverhältnissen/Arbeitsplätzen korrespondiert, wie aus der Erläuterung hervorgeht:

Wir wollen mehr feste Arbeitsplätze.

Das bedeutet: Firma und Mitarbeiter zahlen einen Teil vom Lohn

in die Sozial-Versicherung. 
Sozial-Versicherung ist zum Beispiel die Renten-Versicherung.

Oder die Kranken-Versicherung.

Ein Teil vom Lohn zahlt man für diese Versicherungen.

(SPD-Wahlprogramm LS: 8, Hervorhebungen im Original)

Ob man die Worterklärung von "feste Arbeitsplätze" und "Sozialversicherung" als gelungen bezeichnen kann, sei dahingestellt. Es ist jedoch das einzige Beispiel, in dem ein neues Konzept unter Verwendung eines zumindest ähnlichen Schlagworts (Sozialversicherung - allerdings nicht: sozialversicherungspflichtig) eingeführt wird. Die Passage wird in Kapitel 4.3 noch einmal zur Sprache kommen.

Die Inklusions-bezogenen Schlagworte (Inklusion/inklusiv, Mitwirkung, Mitbestimmung, Selbstbestimmung, Teilhabe ${ }^{16}$ oder Partizipation) werden wie bereits erwähnt in der einfachen Fassung nicht genannt. In der leichten Fassung ebenfalls nicht, allerdings finden die Themen in sehr allgemeiner Weise im gesamten Text ihren Niederschlag, und zwar u. a. in einer bestimmten Ausdrucksweise: Das Indefinitum alle (ohne darauffolgende Nominalphrase, 18 Belege), die Phrase "alle Menschen" (8 Belege) sowie "jeder Mensch" (10 Belege) werden auffällig häufig verwendet, teilweise auch gehäuft:

Jeder Mensch, der arbeitet, soll genug Geld zum Leben haben.

(SPD-Wahlprogramm LS: 8)

Alle haben die gleichen Möglichkeiten. / Alle sollen eine Ausbildung machen können. / [...] Jeder Mensch hat ein Recht auf gute Bildung.

Alle Menschen sollen gut zusammen leben. / Alle sollen gut miteinander umgehen. / Jeder Mensch soll mitmachen können.

(ebd.: 17)

Alle sollen mitmachen können. / [...] Alle können überall mitmachen. / Jeder Mensch lebt mittendrin in der Gesellschaft.

(ebd.: 18)

Jeder Mensch darf selbst bestimmen, wie er wohnen möchte.

(ebd.: 19)

Zusammenfassend kann man also sagen, dass sich die leichte Fassung durch ein konsequentes Vermeiden zentraler Schlagwörter auszeichnet, während die einfache Fassung recht offenkundig das Bemühen erkennen lässt, an solche Wörter und Konzepte anzuschließen und sie zu erklären. Problematisch ist das konsequente Vermeiden, weil der Anschluss an den allgemeinen Diskurs fehlt und vom Leser nicht hergestellt werden kann, wenn er nicht entsprechendes Vorwissen ohnehin schon besitzt. Auch das Verständnis des allgemeinen Sprachgebrauchs wird nicht gefördert. Der Leser lernt also auch sprachlich "nichts dazu", sondern wird in der leichten (Text-)Welt stehen gelassen. Zugespitzt formuliert: Wer nur leichte Texte rezipiert, befindet sich so letztlich in einem reduzierten und isolierten Paralleldiskurs (Stichwort: ZweiWelten-Theorie), dessen Grenzen ohne Hilfe schwer zu überwinden sein dürften. Ein sol-

16 Teilhabe ist ein Schlüsselwort der Vollfassung gegenüber leichter und einfacher Wahlprogrammfassung (Keyness-Wert: 10.735). 
chermaßen gestalteter Text bietet kaum Möglichkeiten (z. B. durch Hinweise auf weiterführende Informationen), das Wissen und (sprachliche und weiterführende) Kompetenzen selbständig zu erweitern. Es geht schließlich nicht nur um den Gebrauch bestimmter Wörter, sondern auch um die damit verbundenen Konzepte. Wer noch nie etwas von Mindestlohn, Tariflohn oder Betriebsrat gehört hat, weil er nur den an seine Zielgruppe adressierten leichten Text kennt, ${ }^{17}$ der kann dies auch schlecht(er) einfordern oder sich dazu positionieren. Für den Bereich Politik kann man festhalten, dass eine gewisse "Schlagwort-Kompetenz" das Verständnis aktueller öffentlicher Debatten und politische Partizipation sicherlich erleichtert.

\subsection{Vagheit}

Die Vagheit von Aussagen im leichten Wahlprogrammtext ist bereits angesprochen worden. Tatsächlich kann man die semantische Unbestimmtheit und Allgemeinheit von Formulierungen als Problem in vielen Leichte Sprache-Texten ausmachen. Ein Teil der Vagheit entsteht durch semantisch relativ unspezifischen Wortschatz. Es gibt eine Tendenz zu Hyperonymen. Ein Beispiel: Ein wichtiges (quantitativ bestimmtes) Schlüsselwort des leichten Wahlprogramms gegenüber der Vollfassung ist Menschen (Keyness-Wert: 98.970). In der Wahlprogramm-Vollfassung wird dieses Lexem zum einen signifikant seltener verwendet, zum anderen wird es aber, wie die Konkordanzanaylse zeigt, auch in den meisten Fällen mit spezifizierenden Attributen oder in formelhaften Wendungen (Menschen in Deutschland/in unserem Land/aller Schichten/mit normalem Einkommen/junge Menschen usw.) verwendet. Statt des Hyperonyms Menschen werden viele spezifischere Personenbezeichnungen benutzt, wie bspw. - je nach Thema - Arbeitnehmer, Migranten, Rentner, Bürger, "Junge und Ältere", Frauen und Männer, Deutsche und Nicht-Deutsche, Gesunde und Kranke, Menschen mit und ohne Behinderung" (SPD-Wahlprogramm VV: 6) - also meistenteils Ausdrücke, die kaum als schwer verständlich gelten können. In der einfachen Wahlprogrammfassung kommt Menschen nicht signifikant häufig vor, es ist also kein Schlüsselwort. Das kann als Indiz für weniger Vagheit in dieser Hinsicht gedeutet werden.

Vagheit entsteht in der leichten Fassung außerdem durch semantisch "blasse" Verben mit wenig spezifischen Bedeutungsanteilen. Das Themenfeld Inklusion wird beispielsweise unter der Überschrift "Alle sollen mitmachen können" verhandelt. Mitmachen ist das zentrale Wort und Paraphrase für Schlagworte wie Partizipation, Inklusion, die wie gesagt ungenannt bleiben.

Darum wollen wir: ${ }^{18}$

- Alle können überall mitmachen.

Jeder Mensch lebt mittendrin in der Gesellschaft.

Niemand wird ausgeschlossen.

\footnotetext{
${ }^{17}$ Im Fall des SPD-Wahlprogramms ist beispielsweise kaum denkbar, dass Menschen mit Lern- oder auch nur Leseschwierigkeiten sich auch durch die 120 Seiten starke und in weiten Teilen stark fach(sprach)liche Vollfassung arbeiten würden oder könnten. In einer Explorationsstudie konnte ich zudem die Erfahrung machen, dass die beiden Probanden bei einem vorgelegten Text, der spaltenweise eine leichte und eine normale Fassung nebeneinander darstellte, ausschließlich die Spalte in Leichter Sprache lasen und die andere Spalte ignorierten (vgl. Bock 2014).

18 Auf die Verständnisschwierigkeiten durch die eigensinnige Verwendung von Konnektoren und anderen Kohäsionsmitteln kann hier nicht eingegangen werden.
} 
Menschen mit Behinderungen machen genauso mit,

wie Menschen ohne Behinderungen.

Überall da, wo man lebt, soll man mitmachen können.

Zum Beispiel:

- In der Kinder-Tages-Stätte

- In Schulen oder an der Uni

- Im Sport-Verein

- In Volks-Hoch-Schulen

(SPD-Wahlprogramm LS: 18)

Die politische Forderung wird mit dem so vagen wie unspezifischen Satz "Alle können überall mitmachen." vorgebracht. Es geht um "mitmachen" "überall da, wo man lebt" in der "Gesellschaft". Besonders die Unterspezifiziertheit des Verbs mitmachen macht den Text (trotz aufgezählter Beispiele!) wenig anschaulich: Wer macht in welcher Rolle wobei in welcher Weise und mit welchem Ziel mit? Framesemantisch ausgedrückt ruft das Wort einen Frame auf, der einen sehr hohen Allgemeinheitsgrad hat, wobei wesentliche kontextspezifische Leerstellen nicht aufgerufen oder gefüllt werden. Es fehlt also eine Konkretisierung. Auch Leser, die eine Verbindung zu Frames wie Inklusion, Teilhabe usw. herstellen, können die Bedeutung von mitmachen hier nur schwer explizieren. Differenzierungen wie die zwischen Mitwirkungs- und Mitbestimmungsrechten ${ }^{19}$ zu treffen oder die genaue Vorstellung der SPD (z. B. die "Reichweite") von Teilhabe und Partizipation zu benennen, ist auf Basis dieser Textfassung letztlich unmöglich. Das in dieser Passage formulierte politische Ziel wird erneut nicht begründet oder argumentativ hergeleitet.

Durch die vage und generalisierende Formulierungsweise entstehen auch Widersprüche zwischen Textpassagen:

In Deutschland leben viele Menschen mit Behinderungen.

Sie haben die gleichen Rechte wie alle anderen Menschen.

Aber viele werden trotzdem benachteiligt.

Zum Beispiel:

Viele Kinder mit Behinderungen sind in einer Sonderschule.

Viele Menschen mit Behinderungen bekommen keinen Arbeitsplatz

in einer Firma.

Das finden wir ungerecht.

Unser gemeinsames Ziel ist:

Menschen mit Behinderungen sollen die gleichen Rechte

wie alle anderen Menschen haben.

Sie dürfen nicht benachteiligt werden.

(SPD-Wahlprogramm LS: 18, Hervorhebungen B. Bock)

Das Modalverb sollen im zweiten hervorgehobenen Satz präsupponiert, dass es (noch) nicht der Fall ist, dass Menschen mit Behinderung die gleichen Rechte wie alle anderen Menschen

19 Vgl. mit Bezug auf die Situation von Menschen mit Lernschwierigkeiten (vgl. Niehoff 2013: 237-238). 
haben (deontische Aussage). Genau das wird aber kurz zuvor als Ist-Zustand beschrieben (deskriptive Aussage). Die angeführten Beispiele für Benachteiligungen sind außerdem keineswegs selbsterklärliche Argumente für den Schluss, dass die jeweils benannte Situation "ungerecht" sei. Dem Schluss liegt ein ganz bestimmtes Verständnis von inklusiver Gesellschaft zugrunde, das aber in der "leichten" Wahlprogrammfassung nirgendwo explizit gemacht wird.

Dem Leser wird in diesem Text einiges an Vorwissen und Inferenzfähigkeiten abverlangt. Die Vagheit und "semantische Armut" vieler Formulierungen (und die häufige Unverbundenheit der Aussagen und mangelnde Kohäsion auf syntaktischer Ebene) führen außerdem zu einer gewissen Monotonie, die kaum lesemotivierend wirken dürfte. Insbesondere im ersten Textausschnitt erhält der Leser kaum substanzielles Wissen über Partizipationsmöglichkeiten. In beiden Passagen werden die Ziele der SPD kaum mit der Absicht dargestellt, den Leser überzeugen oder umfassend informieren zu wollen. In der einfachen Fassung, die die politischen Themen insgesamt anschaulich aufbereitet, fehlt interessanterweise ein thematisch entsprechendes Kapitel.

\section{3 (Fehlende) Sprachhandlungen}

Wiederholt wurde zu den bisher zitierten leichten Textpassagen angemerkt, dass da, wo in einem Wahlprogramm Argumentation und Begründung erwartbar wären, darauf verzichtet wird. Ziele und Forderungen werden stattdessen eher "monolithisch" und konstatierend vorgestellt. So auch in diesem bereits zitierten Ausschnitt:

Wir wollen mehr feste Arbeitsplätze.

Das bedeutet: Firma und Mitarbeiter zahlen einen Teil vom Lohn

in die Sozial-Versicherung.

Sozial-Versicherung ist zum Beispiel die Renten-Versicherung.

Oder die Kranken-Versicherung.

Ein Teil vom Lohn zahlt man für diese Versicherungen.

(SPD-Wahlprogramm LS: 18, Hervorhebungen im Original)

Fasst man die Informationen, die hier der Reihe nach vorgebracht werden, knapp zusammen, wird die Forderung nach "mehr festen Arbeitsplätzen" durchaus missverständlich für Leser, die ein entsprechendes Vorwissen nicht mitbringen. Es steht dort: "Wir wollen mehr feste Arbeitsplätze. Das bedeutet, dass die Firma und die Mitarbeiter einen Teil vom Lohn abgeben müssen." Die Vorteile "fester Arbeitsplätze" werden hingegen nicht explizit erwähnt. Es überrascht, dass hier nicht um Zustimmung geworben, sondern dass das Leseverständnis der Inferenzziehung und dem Weltwissen des individuellen Lesers überlassen wird. In dieser Weise werden etliche Ziele im leichten Wahlprogramm eher postuliert als begründet, und es wird kaum um Zustimmung (und Wählerstimmen) geworben.

Dass man den Zielgruppen Leichter Sprache Argumentation und differenzierende Begründung offenkundig nicht (oder nur eingeschränkt) zutraut, ist im Kommunikationsfeld Politik m. E. nicht unproblematisch. Die Wahlentscheidung und jegliche andere Form der Partizipation setzen voraus, dass der Einzelne nicht nur mit allgemeinen Informationen versorgt wird, sondern ihm die Chance gegeben wird, sich auf der Basis hinreichenden Wissens eine Meinung zu bilden. Das kann freilich nicht bedeuten, dass eine Textsorte wie das Wahlprogramm 
sämtliche für die politische Meinungsbildung notwendige Informations- und Bildungsarbeit leisten kann oder soll. Aber es muss - genau wie bei der Wahlprogrammfassung, die sich an die große Allgemeinheit richtet - wenigstens die Intention erkennbar sein, zentrale Informationen adressatenspezifisch darzustellen und die Leser insoweit ernst zu nehmen, als man ihnen Beweggründe und Argumente für politische Positionen darstellt (Befürwortung und Ablehnung liegen dann immer noch auf Seiten des Lesers). Im Sinne der Barrierefreiheit könnte man daher wieder bildlich bemängeln, dass der Text zu wenig die Intention erkennen lässt, dem Leser "eine Brücke zu bauen". Besonders im Fehlen von Argumentation und Begründung zeigt sich, dass die schon erwähnte Aushängeschild-Funktion vieler leichter Texte hier auch eine Rolle spielt: Man stellt ein Wahlprogramm mit dem Label Leichte Sprache zur Verfügung, versucht darin aber gar nicht, den Leser zu überzeugen oder (anschlussfähig) zu informieren. Ein ernsthaftes Angebot sähe anders aus.

Diese Kritik entspringt keineswegs einer generellen Ablehnung oder Infragestellung der Idee einer zielgruppenzugeschnittenen Verständlich- und Zugänglichmachung von Texten, wie sie Ansätze wie die Leichte Sprache ja verfolgen. Die kritische Kommentierung ist eher auf die Umsetzung, auf die gängige Praxis gerichtet. Dass Verständlichmachung durchaus "Barrierensenkung" bedeuten kann, wenn sie nicht eindimensional auf Reduzierung (von sprachlichen Ausdrucksmöglichkeiten und inhaltlichem Gehalt) setzt, zeigt nämlich die einfache Fassung des SPD-Wahlprogramms: Dort wird begründet und argumentiert, dort werden Zusammenhänge zwischen Teilthemen ausdrücklich hergestellt, außerdem wird viel mit grafischen Veranschaulichungen gearbeitet. Für welche Zielgruppen eine solche Aufbereitung geeignet ist und wo sie ggf. doch an Verstehensgrenzen bei Lesern stößt, ist empirisch noch zu überprüfen. Positiv zu verzeichnen ist aber schon jetzt, dass der einfache Text in angemessener Weise die Textfunktionen umzusetzen versucht, die der Textsorte Wahlprogramm entsprechen, und dass er den Anschluss an den allgemeinen Diskurs sucht (bspw. durch Erklärung zentraler Schlagwörter). Ein solches Bewusstsein für Verständlichkeitsaspekte lässt sich bei der leichten Fassung nicht in gleichem Maße erkennen.

\subsection{Ein Ausdruck - zwei Strategien}

In einer direkten Gegenüberstellung von leichter, einfacher und Wahlprogramm-Vollfassung sollen Unterschiede im semantischen Gehalt und der Darstellungsweise herausgearbeitet werden, und zwar am Beispiel eines Ausdrucks - Ganztagsschule -, der in allen Texten verwendet wird. Interessant ist hier, wie selbst bei einem anscheinend einfachen und konkreten Beispielwort unterschiedliche Bedeutungsgehalte aktualisiert werden. Es zeigt sich außerdem Vieles von dem, was in den vorangegangenen Kapiteln an unterschiedlichen Beispielen herausgearbeitet wurde.

In der Vollversion des Wahlprogramms taucht der Begriff Ganztagsschule im Kapitel "Bildung, Gleichberechtigung und Zusammenleben in einer modernen Gesellschaft" auf, und zwar im Kontext der Kapitel "Chancengleichheit und Aufstieg durch Bildung" und "Familien gehen vor". Schon mit den Überschriftentiteln deutet sich an, dass Ganztagsschule weniger als Konkretum gebraucht, erklärt und vorgestellt wird als vielmehr im Zusammenhang abstrakterer politischer Ziele. Die erste Nennung des Wortes findet sich dann auch in dem Kompositum Ganztagsschulprogramm (SPD-Wahlprogramm VV: 7). Tatsächlich erfolgt die Thematisierung - anders als in der leichten und einfachen Fassung - vorrangig im Kontext von 
abstrakten Frames wie 'Sozialstaat', 'Chancengleichheit/Bildungschancen', 'inklusive Bildung' und 'materielle Absicherung'. Erklärt wird Ganztagsschule als "Erfolgsmodell, das mehr Zeit zum Lernen bietet" (ebd.: 43), außerdem werden die "Möglichkeiten für die gezielte Förderung für jede und jeden Einzelnen und für den Umgang mit heterogenen Lerngruppen" hervorgehoben (ebd.). Indem die Zeit zum Lernen, indem Förderung und heterogene Lerngruppen erwähnt werden, werden einzelne Leerstellen des Frames 'Institution (Ganztags-)Schule' kontextabhängig gefüllt. Diese Fillers sind im Text positiv besetzt. Konkretisierungsansätze sind rar: Das Konzept Ganztagsschulen wird an keiner Stelle aus einer alltagsbezogenen Perspektive thematisiert oder explizit auf die Lebenswelt potenzieller Leser bezogen und so konkretisiert.

Folgt man den Klassifizierungen in FrameNet, ist der Ausdruck "Schule" (und damit auch Ganztagsschule) in drei übergeordnete Frames einordenbar: Aggregate (Ansammlung von Individuen), Locale_by_Use (Ort mit einer bestimmten Funktion), Education_teaching (Bildung). In der Vollversion des Wahlprogramms werden die beiden konkreteren Frames (Aggregate, Locale_by_Use) nicht unmittelbar aktualisiert. Stattdessen wird eher der allgemeine Matrixframe 'Bildung' aufgerufen, in dem die 'Institution' (z. B. Schule) eines der semantischen Kernelemente darstellt. Die Thematisierung erfolgt im Rahmen übergeordneter, abstrakter Frames wie 'Bildungspolitik' oder 'Sozialpolitik'.

Leichte und einfache Fassung des SPD-Wahlprogramms setzen den Ausdruck Ganztagsschule demgegenüber in konkrete Kontexte. In der leichten Fassung wird das Wort erklärt:

Dort können die Kinder den ganzen Tag bleiben.

Sie haben mehr Zeit, um miteinander zu lernen.

(SPD-Wahlprogramm LS: 9)

Hier wird der Frame 'Ort mit einer bestimmten Funktion' aktualisiert. Als semantische Kernelemente gibt FrameNet 'Ort' und (nicht immer ausgedrückt) 'Funktion' an. Die Leerstelle 'Funktion' (von Ganztagsschulen) wird im Text gefüllt durch die Aussage 'Zeit haben, um miteinander zu lernen'. Außerdem wird eine weitere Leerstelle aufgerufen und gefüllt, die im Kontext von Ganztagsschulen relevant ist: die Aufenthaltsdauer ("den ganzen Tag"). Der knappen Textpassage voraus gehen Abschnitte, in denen erläutert wird, was die SPD unter guter Bildung (für alle) versteht. Unter der darauffolgenden Überschrift "Darum wollen wir:" wird dann als erstes Ziel "Mehr Ganz-Tags-Schulen" lediglich genannt. Eine Begründung oder Erläuterung dieses Ziels fehlt.

In der Broschüre in einfacher Sprache wird das Konzept Ganztagsschule ebenfalls in konkrete Kontexte gesetzt. Der Unterschied ist, dass mehr Bedeutungselemente realisiert werden als in der leichten Version des Wahlprogramms. Erklärt wird das Wort in der Randspalte folgendermaßen:

Dort können die Kinder bis zum späten Nachmittag in der Schule bleiben. Sie haben nicht nur Unterricht. Je nach der Art der Ganztagsschule gibt es dort auch Freizeit-Angebote oder Begleitung bei den Hausaufgaben.

(SPD-Wahlprogramm ES: 11)

Außerdem wird das Ziel "Mehr Ganztagsschulen" im Text begründet: 
Wenn Kinder miteinander lernen, lernen sie schneller und besser. Darum soll es mehr Ganztagsschulen geben. Eltern können gleichzeitig Kinder und Beruf besser miteinander vereinbaren.

(ebd.)

Im einfachen Wahlprogramm wird also zunächst einmal auch der Frame 'Ort mit einer bestimmten Funktion' aufgerufen, und der Text füllt die Leerstellen Funktion und Aufenthaltsdauer. Diese Füllung ist jedoch sowohl spezifischer als auch umfassender als im leichten Text: Statt der unspezifischen Angabe den ganzen Tag wird die Aufenthaltsdauer hier mit "bis zum späten Nachmittag" angegeben; noch interessanter ist aber, dass als Zweck nicht nur Lernen ("Sie haben nicht nur Unterricht"), sondern auch andere Aufgaben genannt werden, nämlich Freizeitangebote und Hausaufgabenbegleitung. Hier wird deutlich, dass die leichte Fassung den Ausdruck Ganztagsschule zwar genau wie die Broschüre in einfacher Sprache auf den konkreten Erfahrungsraum potenzieller Leser bezieht, aber deutlich weniger Bedeutungsaspekte aktualisiert. Bei diesem (eigentlich nicht sehr komplexen) Beispiel führt das sogar dazu, dass die leichte Worterklärung sachlich nicht ganz richtig ist, denn in Ganztagsschulen geht es eben nicht nur um gemeinsames Lernen den ganzen Tag. Ob eine so geartete Einrichtung potenziellen Wählern als attraktiv erscheint, bleibt außerdem fraglich.

Die Hauptprobleme des Wahlprogramms in Leichter Sprache, wie sie am Beispiel des Ausdrucks Ganztagsschule deutlich werden, sind also framesemantisch ausgedrückt die Allgemeinheit bzw. Unterspezifiziertheit der im Text realisierten Füllwerte sowie deren reduzierte Zahl. An solchen Stellen wird die viel zu ausschließlich auf Reduktion angelegte Verständlichmachungs-Strategie vieler Texte deutlich. Ebenfalls problematisch ist, dass Ganztagsschulen nie in einem Begründungszusammenhang thematisiert werden. Obwohl als Textsortenbezeichnung explizit "Wahlprogramm (in Leichter Sprache)" angegeben wird und die Darstellung von "Zielen der SPD" den Text strukturiert, kann man zu dem Schluss gelangen, dass die leichte Fassung - exemplarisch untersucht am Beispiel Ganztagsschule und den Kapiteln zur Arbeitspolitik - den zentralen Funktionen von Wahlprogrammen kaum gerecht wird: Informationsfunktion hat der Text sicherlich, mit der Appell- bzw. Werbefunktion wird es allerdings schon schwierig. Das für Wahlkampf-Werbeschriften eigentlich konstitutive

Konglomerat [...] aus Thesen, Behauptungen, Forderungen, Beschuldigungen, Ankündigungen und Bewertungen, die mehr oder weniger argumentativ untermauert werden, und zwar aus einseitig parteilicher Perspektive, d. h. mit durchgängiger Aufwertung eigener und Abwertung gegnerischer Positionen

(Klein 2014: 171)

ist in dieser Variationsbreite nicht ansatzweise zu finden. Politische Positionen werden teilweise nicht einmal deutlich herausgearbeitet. Für welche Leser sind solche Texte gedacht?

Die einfache Fassung des Wahlprogramms realisiert die textsortentypischen Funktionen besser: Bereits in den äußerst knappen Passagen zum Thema Ganztagsschule (vgl. oben) wird argumentiert und begründet. Vorteile der jeweiligen politischen Position werden deutlich gemacht. Die einzelnen Ziele werden nicht nur genannt (Informationsfunktion), sondern es werden auch Bedingungen oder Grund-Folge-Beziehungen formuliert, die den Leser argumentativ überzeugen sollen (Appellfunktion). Diese Beziehungen werden häufig explizit sprachlich ausgedrückt, d. h. das Verständnis beim Leser wird unterstützt und vereindeutigt: In den oben zitierten Passagen zur Ganztagsschule werden eine Reihe von Textverknüpfungsmitteln gebraucht, die die jeweiligen Kohärenzrelationen unmittelbar abbilden (die Konnektoren darum, 
weil, wenn). Zudem werden Aussagen anaphorisch eng verknüpft (durch dort) und damit das in der Leichten Sprache verbreitete Prinzip der Wiederaufnahme durch Rekurrenz zugunsten größerer Formulierungsvariation durchbrochen. Die Sätze sind genau wie in der leichten Fassung sehr kurz gehalten. Es dominieren Parataxen; die für die Leichte Sprache typischen Ellipsen kommen hier ebenfalls vor.

Eine Darstellungsstrategie in der einfachen Fassung des Wahlprogramms besteht $u$. a. in Veranschaulichung durch Bezug auf den konkreten Erfahrungsraum potenzieller Leser. Damit ist natürlich ebenfalls eine Reduktion der in der Wahlprogramm-Vollfassung aufgerufenen Bedeutungselemente verbunden, aber keine solche Unterspezifiziertheit und Vagheit wie häufig in der leichten Fassung. Nach der Untersuchung der (zugegebenermaßen begrenzten Anzahl von) Textstellen könnte man vielleicht zusammenfassend sagen, dass in der einfachen Wahlprogrammfassung zwar reduziert wird, dass aber die Darstellung dessen, was nicht weggelassen wird, durchaus vielschichtig und der Textfunktion und der Textsorte angemessen erfolgt. Nur wenn das gewährleistet ist, kann man m. E. davon sprechen, dass ein Text ein Beitrag zur Barrierefreiheit ist und Partizipation (mit) ermöglichen kann.

\section{$5 \quad$ Perspektiven}

Auch andere Arten barrierefreier Kommunikation haben mit dem Problem inhaltlicher Selektion $\mathrm{zu}$ tun sowie mit der Herausforderung, Texte so $\mathrm{zu}$ modifizieren (medial, sprachlich, inhaltlich), dass sie der jeweiligen kommunikativen Aufgabe und Situation angemessen sind. Die Ausgangslagen sind verschieden: Bei Braille-Schrift können (Schriftsprach-)Informationen nahezu vollständig äquivalent in ein anderes Medium übertragen werden, die Umsetzung von Bildinformationen für den Sehenden bedarf allerdings der Modifikation in Sprache. Beim Respeaking, der Live-Untertitelung als Einsprechen schriftlich ausgegebener Untertitel, muss aufgrund des Zeitdrucks gekürzt, gerafft und umformuliert werden (vgl. Jekat 2014). Konnotative, stilistische und auch sachbezogene Informationen gehen dabei durchaus verloren. Allerdings sind es professionelle Übersetzer, die Respeaking umsetzen. Für den Bereich "Leichte Sprache" fehlt eine vergleichbare flächendeckende Professionalisierung bisher. Bei der Audiodeskription (bspw. Hörfilme, vgl. Poethe 2005) für Sehgeschädigte findet ebenfalls eine inhaltliche Selektion statt, denn nicht alle visuellen Informationen können verbalisiert werden. Die Mittel der Audiodeskription werden allerdings im Gegensatz zur Leichten Sprache schon lange in Theorie und Praxis ausführlich reflektiert: Als Ziel formuliert beispielsweise Benecke (2014: 110) die "Vermittlung eines erlebten Eindrucks"; die relevanten Bildinformationen sollen abhängig von ihrer Relevanz möglichst weitgehend zugänglich gemacht werden. Derzeitige Texte in Leichter Sprache zeigen demgegenüber eine allzu einseitige Tendenz zu Reduzierung, Selektion und Vereinfachung.

Diese Gegenüberstellung soll deutlich machen, dass die unterschiedlichen Formen barrierefreier Kommunikation teilweise vor ähnlichen Herausforderungen stehen. Dennoch sind die Situationen unterschiedlich. Durch die ausgeprägte (und schwer vollständig auflösbare) Asymmetrie zwischen Texterstellern und Zielgruppe ist das beschriebene Risiko, lediglich Zugang zu einer Parallelwelt aus angepassten Texten zu ermöglichen und nur einen rudimentären Bezug zur allgemeinen Welt herzustellen (und damit Partizipation zu ermöglichen), bei Leichter Sprache größer als bei anderen Formen barrierefreier Kommunikation. Die wissen- 
schaftliche Begleitung und Weiterentwicklung des Ansatzes beginnt gerade erst, obwohl er in der Praxis in der derzeitigen Form schon als etabliert gelten kann. Nicht zuletzt die (sehr heterogene) Zielgruppe stellt eine Herausforderung für die Ausarbeitung eines einheitlichen Konzepts dar. Dennoch gibt es in der Angewandten Linguistik bereits Forschung (Verständlichkeitsforschung, Textoptimierung für Hörgeschädigte), die bisher nicht in die Ausarbeitung des Konzepts eingeflossen ist. Auch in der Praxis gibt es - wie die Analyse zeigen konnte durchaus Beispiele für gelungene Modifizierungen in eine leichter verständliche Form. Es bleibt fraglich, ob die derzeit in Regelkatalogen (Netzwerk Leichte Sprache 2013) aufgelisteten, kontextunspezifisch formulierten Leichte-Sprache-Prinzipien eine optimale und angemessene Textmodifizierung ermöglichen. ${ }^{20}$

Wichtig für eine Weiterentwicklung des Ansatzes ist zum einen, dass Möglichkeiten der Verständlichmachung textsorten- und kommunikationsbereichsspezifisch erforscht und ausdifferenziert werden, und zum anderen, dass nicht nur über die sprachliche (und typografische) Oberfläche, sondern auch über die Inhalte der Texte gesprochen wird. Inhaltliche Komplexität kann nicht durch sprachliche Einfachheit "aufgelöst" werden. Bei jeder Modifizierung werden auch die Inhalte verändert, was die m. E. bisher nicht beantwortete Frage aufwirft, wozu die Leser leichter Texte (bspw. der beiden untersuchten Wahlprogrammfassungen) befähigt werden sollen, was sie nach der Lektüre wissen und können sollen. ${ }^{21}$ Simone Seitz (2014: 5) bezeichnet die Erstellung leichter Texte daher auch zu Recht als "fachlich anspruchsvolle didaktische Aufgabe", bei der es darum ginge, "Zugänge zu komplexen Sachzusammenhängen zu ermöglichen". Meines Erachtens muss die Vermittlungsaufgabe, die Leichter Sprache unzweifelhaft zukommt, sowohl in der Praxis als auch in der theoretischen Modellierung des Ansatzes stärker Berücksichtigung finden: Versteht man Leichte Sprache konsequent als Vermittlungsvarietät, die Barrieren senken und Zugang zu auch komplexen Texten und Inhalten verschaffen soll, dann rückt auch die Aufgabe, die Kompetenzentwicklung auf Seiten der Leser zu fördern bzw. überhaupt zu ermöglichen, ins Blickfeld. D. h. leichte Texte müssen zum einen Inhalte adressaten-, situations- und gegenstandsangemessen aufbereiten, sodass Verständnis und Anschlusshandlungen für die Zielgruppen möglich sind. Zum anderen muss der Blick stärker auf die Aufgaben der Entwicklung von sprachlichen, lebenspraktischen usw. Kompetenzen gerichtet werden. Schwierigkeitsstufen leichter Texte wären hier ein Teilziel. ${ }^{22}$

\section{$6 \quad$ Fazit}

Leichte Sprache ist ein Mittel, um Partizipationschancen von Menschen mit Lernschwierigkeiten zu verbessern. Dazu muss aber das Ziel der Absenkung von (Kommunikations-)Barrieren (Barrierefreiheit) konsequenter umgesetzt werden: Leichte Sprache muss ihren Status als Vermittlungsvarietät (vgl. Bock i. E.), und damit ihre Brückenfunktion ernster nehmen und konsequenter in den Texten verwirklichen. Dann können leichte Texte dazu beitragen, politische Partizipation für die Zielgruppe zu

\footnotetext{
${ }^{20}$ Die einzelnen Textexemplare halten sich häufig ohnehin nicht daran.

21 Darüber müssen sich bereits die Textersteller Klarheit verschaffen.

22 Zwar gibt es von Agenturen bereits Differenzierungen in Schwierigkeitsstufen, diese lehnen sich aber meist an den Gemeinsamen Europäischen Referenzrahmen und damit an das Erlernen von Fremdsprachen an. Ob dies ein aussichtsreicher Ansatz ist, bleibt in Anbetracht der spezifischen Eigenschaften der Zielgruppe zu überprüfen.
} 
ermöglichen bzw. die Schwelle dahin zu senken und den Weg zu mehr Selbstbestimmung zu ebnen.

In der Analyse der Wahlprogrammfassungen sind keineswegs alle Aspekte angesprochen worden, die Verständlichkeit und Barrierensenkung beeinflussen. Es wurden exemplarisch positive und kritikwürdige Prinzipien der Textgestaltung nachvollzogen und die Folgen für die Partizipation der angesprochenen Zielgruppe beschrieben. Die Illusion eines reinen Umschaltens der sprachlichen Oberfläche auf leicht verständlich hat sich dabei als ebenso wenig förderlich herausgestellt, wie die inhaltliche und sprachliche Vagheit von Textpassagen und die Verwendung einer Art "Sonderwortschatz" unter Vermeidung üblicher Schlagwörter. Man muss auch die Frage stellen, wie ernst die Parteien und Institutionen ihre eigenen Informationsangebote überhaupt nehmen: Denn im Bereich Politik gibt es bereits relativ viele Informationsmaterialien in Leichter Sprache. Diese bieten aber nicht immer ein vollständiges und äquivalentes Informationsangebot, sondern tragen - insbesondere auf den Webseiten von Politikern und Bundesinstitutionen - nur allgemeine Meta-Informationen zusammen (vgl. Bock in Vorbereitung). In solchen Fällen werden die Vermittlungsfunktion und die Aufgabe, Partizipation und Selbstbestimmung durch Barrierefreiheit zu ermöglichen, unterlaufen.

In der Zukunft werden auch die Grenzen der Verständlichmachung für die Zielgruppe zu diskutieren sein. Gilt hier, dass die Grenzen der Barrierensenkung die Grenzen der Partizipation markieren? Partizipation für benachteiligte Zielgruppen ist in jedem Fall nicht nur aus sprachund kommunikationsbezogener Sicht zu behandeln, aber Sprache und Kommunikation spielen eine bedeutende Rolle.

\section{Literatur}

Aus Politik und Zeitgeschichte (2014): Leichte und Einfache Sprache. 9-11/64.

Benecke, Bernd (2014): "Der Ton macht die Audiodeskription. Über Erleichterungen und Herausforderungen durch den Ton des Ausgangsmediums einer Audiodeskription und die Ton-Wahrnehmung von Blinden und Sehbehinderten". In: Jekat, Susanne J. et al. (Hrsg.): Sprache barrierefrei gestalten: Perspektiven aus der Angewandten Linguistik. Berlin, Frank/Timme: 109-126.

Bock, Bettina M. (2014): "'Leichte Sprache'. Abgrenzung, Beschreibung und Problemstellungen aus Sicht der Linguistik". In: Jekat, Susanne J. et al. (Hrsg.): Sprache barrierefrei gestalten: Perspektiven aus der Angewandten Linguistik. Berlin, Frank/Timme: 17-52.

Bock, Bettina M. (2015): "Interaktivität und Multimodalität? Webseiten in 'Leichter' und 'einfacher Sprache"'. In: trans-kom 8/1: 79-102. www.trans-kom.eu/bd08nr01/transkom_08_01_04_Bock_Leichte_Texte.20150717.pdf[15.02.2015].

Bock, Bettina M. (- (i. E.): "Anschluss ermöglichen und die Vermittlungsaufgabe ernst nehmen: 8 Thesen zur 'Leichten Sprache'". Didaktik Deutsch 1/2015.

Bora, Alfons (2005): "'Partizipation' als politische Inklusionsformel". In: Gusy, Christoph/Haupt, Heinz-Gerhardt (Hrsg.): Inklusion und Partizipation. Politische Kommunikation im historischen Wandel. Frankfurt a. M./New York, Campus: 15-34.

DVfR/DGRW (Hrsg.) (2012): Diskussionspapier Teilhabeforschung. www.dvfr.de/fileadmin/download/Fachaussch\%C3\%BCsse/Forschung/Diskussionspapier_ Teilhabeforschung_-_DVfR-DGRW_M\%C3\%A4rz2012.pdf [07.02.2015].

Feilke, Helmuth (2012): "Bildungssprachliche Kompetenzen - fördern und entwickeln". Praxis Deutsch 233/05: 4-13.

Gusy, Christoph/Haupt, Heinz-Gerhard (Hrsg.) (2005): Inklusion und Partizipation. Politische Kommunikation im historischen Wandel. Frankfurt a. M./New York: Campus. 
Jäger, Ludwig (1996): "Expertenkultur und Sprachkultur: 'Innersprachliche Mehrsprachigkeit' und das Problem der Transparenz des Expertenwissens". In: Kerner, Max (Hrsg.): Aufstand der Laien. Expertentum und Demokratie in der technisierten Welt. Aachen/Leipzig/Paris: Thouet.

Jekat, Susanne J. (2014): "Respeaking: Syntaktische Aspekte des Transfers von gesprochener Sprache in geschriebene Sprache". In: Jekat, Susanne J. et al. (Hrsg.): Sprache barrierefrei gestalten: Perspektiven aus der Angewandten Linguistik. Berlin, Frank/Timme: 87-108.

Jekat, Susanne J. et al. (Hrsg.) (2014): Sprache barrierefrei gestalten: Perspektiven aus der Angewandten Linguistik. Berlin: Frank/Timme.

Klein, Josef (2014): "Textsorten im Bereich politischer Institutionen". In: Klein, Josef: Grundlagen der Politolinguistik. Ausgewählte Aufsätze. Berlin, Frank/Timme: 151-196.

Niehoff, Ulrich (2013): "Mitbestimmung, Mitwirkung". In: Theunissen, Georg/Kulig, Wolfram/Schibort, Kerstin (Hrsg.) (2013): Handlexikon Geistige Behinderung. Schlüsselbegriffe aus der Heil- und Sonderpädagogik, Sozialen Arbeit, Medizin, Psychologie, Soziologie und Sozialpolitik. Stuttgart, Kohlhammer: 237-239.

Poethe, Hannelore (2005): "Audiodeskription - Entstehung und Wesen einer Textsorte". In: Fix, Ulla (Hrsg.): Hörfilm. Bildkompensation durch Sprache. Berlin, Schmidt: 33-48.

Schröter, Melani/Carius, Björn (2009): Vom politischen Gebrauch der Sprache. Wort, Text, Diskurs. Frankfurt a. M. etc.: Lang. (=Leipziger Skripten 5).

Schubert, Klaus/Klein, Martina (2011): Das Politiklexikon. 5., aktualisierte und erweiterte Auflage. Bonn: Dietz.

Seitz, Simone (2014): "Leichte Sprache? Keine einfache Sache". Aus Politik und Zeitgeschichte 9-11/64: 3-6.

Theunissen, Georg/Schwalb, Helmut (2012): "Von der Integration zur Inklusion im Sinne von Empowerment". In: Theunissen, Georg/Schwalb, Helmut (Hrsg.): Inklusion, Partizipation und Empowerment in der Behindertenarbeit. Stuttgart, Kohlhammer: 11-38.

Theunissen, Georg/Kulig, Wolfram/Schibort, Kerstin (Hrsg.) (2013): Handlexikon Geistige Behinderung. Schlüsselbegriffe aus der Heil- und Sonderpädagogik, Sozialen Arbeit, Medizin, Psychologie, Soziologie und Sozialpolitik. Stuttgart: Kohlhammer.

Wansing, Gudrun (2005): Teilhabe an der Gesellschaft. Menschen mit Behinderung zwischen Inklusion und Exklusion. Wiesbaden: VS Verlag für Sozialwissenschaften.

WHO-ICF (2005): Internationale Klassifikation der Funktionsfähigkeit, Behinderung und Gesundheit. Hrsg. v. Deutschen Institut für Medizinische Dokumentation und Information. www.dimdi.de/dynamic/de/klassi/downloadcenter/icf/endfassung/icf_endfassung-2005-1001.pdf [07.02.2015].

\section{Korpusverzeichnis}

LS-Wahlprogramm. Das Wahl-Programm der SPD für die Bundestags-Wahl in Leichter Sprache. www.spd.de/linkableblob/103836/data/20130628_regierungsprogramm_ 2013_2017_leichte_sprache.pdf [07.02.2015].

ES-Wahlprogramm. Das Wir entscheidet. Kurz und klar: Was wir wollen. www.spd.de/linkableblob/104494/data/broschuere_in_einfache_sprache.pdf [07.02.2015].

VV-Wahlprogramm. Das Wir entscheidet. Das Regierungsprogramm 2013-2017. www.spd.de/linkableblob/96686/data/20130415_regierungsprogramm_2013_2017.pdf [07.02.2015].

Netzwerk Leichte Sprache (2013): Die Regeln für Leichte Sprache. www.leichtesprache.org/images/Regeln_Leichte_Sprache.pdf [07.02.2015].

Stengel, Eckhard (2015): Das Parlament: Missratene Ausgabe in Leichter Sprache über die Charlie-Anschläge. http://meedia.de/2015/01/23/das-parlament-missratene-ausgabe-inleichter-sprache-ueber-die-charlie-anschlaege/ [07.02.2015]. 
NZZ. "Schlimmer als Realsatire". Interview mit Rainer Bremer. In: Neue Zürcher Zeitung (08.09.2014).

www.nzz.ch/wissenschaft/bildung/schlimmer-als-realsatire-1.18378993 [07.02.2015].

Liessmann, Konrad Paul (2014): Analphabetismus als geheimes Bildungsziel. Frankfurter Allgemeine Zeitung. www.faz.net/aktuell/feuilleton/forschung-und-lehre/schlechterechtschreibung-analphabetismus-als-ziel-13167836.html [07.02.2015].

FrameNet. https://framenet.icsi.berkeley.edu/fndrupal/ [07.02.2015]. 